\title{
The Quest for the Ideal Base: Rational Design of a Nickel Precatalyst Enables Mild, Homogeneous $\mathrm{C}-\mathrm{N}$ Cross-Coupling
}

\author{
Richard Y. Liu, ${ }^{\dagger}$ Joseph M. Dennis, ${ }^{\dagger}$ and Stephen L. Buchwald* \\ Department of Chemistry, Massachusetts Institute of Technology, Cambridge, Massachusetts 02139, United States
}

\begin{abstract}
Palladium-catalyzed amination reactions using soluble organic bases have provided a solution to the many issues associated with heterogeneous reaction conditions. Still, homogeneous $\mathrm{C}-\mathrm{N}$ cross-coupling approaches cannot yet employ bases as weak and economical as trialkylamines. Furthermore, organic base-mediated methods have not been developed for $\mathrm{Ni}(0 / \mathrm{II})$ catalysis, despite some advantages of such systems over analogous Pd-based catalysts. We designed a new air-stable and easily prepared Ni(II) precatalyst bearing an electron-deficient bidentate phosphine ligand that enables the cross-coupling of aryl triflates with aryl amines using triethylamine (TEA) as base. The method is tolerant of sterically-congested coupling partners, as well as those bearing baseand nucleophile-sensitive functional groups. With the aid of density functional theory (DFT) calculations, we determined that the electron-deficient auxiliary ligands decrease both the $\mathrm{p} K_{\mathrm{a}}$ of the Ni-bound amine and the barrier to reductive elimination from the resultant $\mathrm{Ni}(\mathrm{II}$ )-amido complex. Moreover, we determined that precluding Lewis acid-base complexation between the Ni catalyst and the base, due to steric factors, is important for avoiding catalyst inhibition.
\end{abstract}

\section{INTRODUCTION}

The development of metal-catalyzed carbon-nitrogen $(\mathrm{C}-\mathrm{N})$ bond-forming reactions has had a transformative impact on the synthesis of pharmaceuticals, agrochemicals, organic materials and fine chemicals. ${ }^{1}$ Catalysts based on palladium and copper have been broadly employed to facilitate the crosscoupling of aryl (pseudo)halides with amine nucleophiles, but these reactions have traditionally required the addition of inorganic bases. ${ }^{2}$ In recent years, however, there has been increased interest in the use of soluble organic bases in place of these commonly used inorganic reagents. ${ }^{3}$ These single-phase reactions are easily transferrable to high-throughput reaction screening settings, continuous flow chemistry, and microfluidic screening platforms. ${ }^{4}$ Moreover, the use of weak organic bases avoids functional group incompatibility issues associated with nucleophilic alkoxide and metal amide bases, especially in combination with amines. ${ }^{5}$ Previously, several phosphazene, ${ }^{6}$ guanidine, ${ }^{6}$ amidine, ${ }^{7}$ and alkyl amine ${ }^{8}$ bases have been shown to facilitate $\mathrm{Pd}$ - and $\mathrm{Cu}-$ catalyzed $^{9} \mathrm{C}-\mathrm{N}$ bond formation. The weakest among these, alkyl amine bases stand out as an attractive class of reagents, particularly since their steric properties, nucleophilicity, and basicity can be precisely tuned. ${ }^{10}$ Furthermore, many trialkylamine reagents, including triethylamine (TEA), are produced on large scale directly from alcohols and ammonia, ${ }^{11}$ making them as inexpensive as common organic solvents.

Previously, our research group demonstrated that a bulky, electron-deficient $\mathrm{Pd}$ catalyst can facilitate $\mathrm{C}-\mathrm{N}$ bond formation in the presence of 1,8-diazabicyclo[5.4.0] undec-7ene (DBU). ${ }^{12}$ Mechanistic investigations ${ }^{13}$ and in-depth reaction optimization studies $^{14}$ suggested that other organic bases, including TEA and diisopropylethylamine (DIPEA, Hünig's base) could facilitate the cross-coupling of aryl triflates and anilines, albeit with
(A) Traditional Methodologies: Inorganic Bases

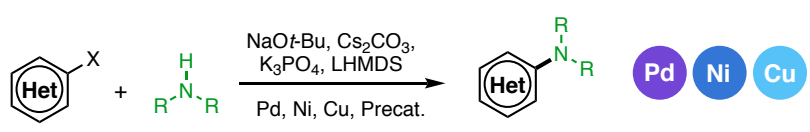

(B) Soluble Organic Bases: Mid-to-High cost; External stimulus (Ni-catalyzed)

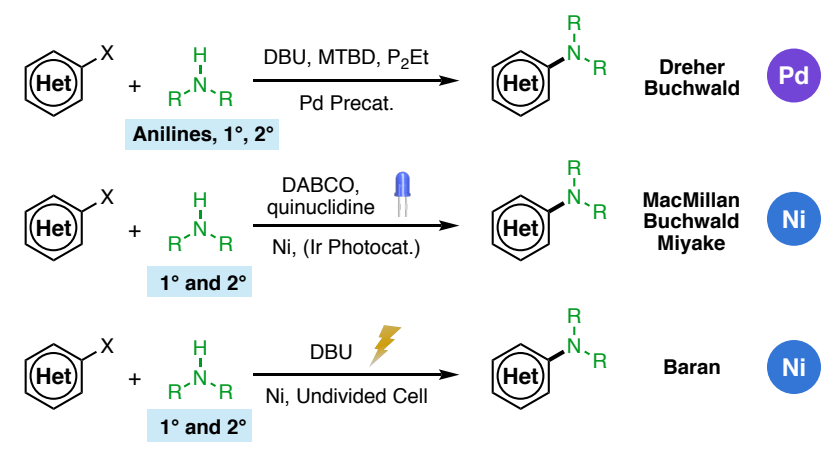

(C) Soluble Organic Bases: Low cost; No external stimulus (This Work)

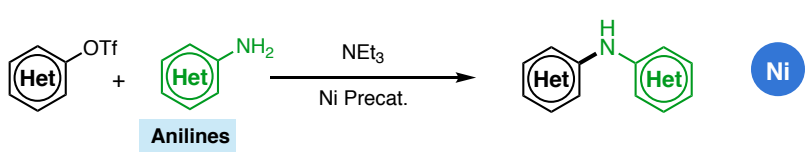

Figure 1. (A) Inorganic bases used in traditional Pd-, Ni-, and $\mathrm{Cu}$-catalyzed $\mathrm{C}-\mathrm{N}$ cross-coupling methodologies. (B) Amidine, guanidine, and phosphazene bases used in $\mathrm{Pd}-$ catalyzed amination and Ni-catalyzed photo- or electrocatalysis. (C) Nickel-catalyzed $\mathrm{C}-\mathrm{N}$ cross coupling of aryl triflates and amines facilitated by triethylamine. 
slower reaction rates. We considered whether an electrondeficient catalyst based on nickel might allow for these very mild and inexpensive trialkylamine bases to be used more effectively in $\mathrm{C}-\mathrm{N}$ cross-coupling.

The use of Ni was of particular interest to us because the use of weak, soluble organic bases in Ni-catalyzed aryl amination has not yet been systematically explored. Since the first reports of Ni-catalyzed amination, ${ }^{15}$ the transformation has been significantly improved in terms of scope and efficiency through rational ligand design, ${ }^{16}$ the development of photocatalytic variants, ${ }^{17}$ and using electrochemistry. ${ }^{18}$ While these efforts have greatly expanded the number and type of electrophiles $^{19}$ and nucleophiles ${ }^{20}$ that can be cross-coupled under practical conditions, the majority of Ni-catalyzed methods remain predominantly reliant on inorganic bases such as metal tert-butoxides and phosphates to facilitate $\mathrm{C}-\mathrm{N}$ formation (Figure 1A). Many useful solutions that are compatible with organic bases take advantage of energy input through either photo- or electrocatalysis. These protocols are primarily limited to the coupling of strongly coordinating nucleophiles such as aliphatic amines (Figure 1, B). ${ }^{17,18}$ Providing a complementary approach, we herein describe the rational discovery of a $\mathrm{Ni}$ (pre)catalyst capable of effecting arylation of weakly binding aniline nucleophiles using a trialkylamine base.

\section{RESULTS AND DISCUSSION}

Our studies began with an evaluation of commercially available bidentate phosphine ligands and organic bases in a model transformation, the Ni-catalyzed cross-coupling of phenyl triflate (1) and aniline. Selected results from these studies are summarized in Table 1 (see Supporting Information for further experimental details). When we used $\mathrm{Ni}(\mathrm{COD})_{2}(4$ mol\%) and 1,1'-bis(diphenylphosphino)ferrocene (L1, DPPF) as precatalysts and triethylamine (TEA) as base, a $6 \%$ yield of the desired product was observed, with unreacted $\mathbf{1}$ making up the remainder of the mass balance. As in our previous work on Pd-catalyzed amination, we predicted that a more electrondeficient metal center would better facilitate the deprotonation of an amine-bound Ni complex by a base as weak as TEA. ${ }^{12 a}$ Accordingly, we prepared several DPPF derivatives bearing electron-withdrawing trifluoromethyl $\left(-\mathrm{CF}_{3}\right)$ substituents on the $P$-aryl groups. ${ }^{21}$ Indeed, use of the fourfold trifluoromethylated ligand $\mathbf{L} 2\left(\left[\mathrm{CF}_{3}\right]_{4}-\mathrm{DPPF}\right)$ resulted in $32 \%$ yield of the desired product. The yield was further increased to $94 \%$ by employing the further trifluoromethylated ligand L3 $\left(\left[\mathrm{CF}_{3}\right]_{8}\right.$-DPPF $){ }^{22}$ The ferrocene backbone was also found to be important to the success of these reactions: other ligands containing similar trifluoromethylated aryl groups, such a 1,2bis(diphenylphosphino)benzene (DPPBz) derivative (L5, $\left.\left[\mathrm{CF}_{3}\right]_{8}-\mathrm{DPPBz}\right)$ were less effective in promoting the $\mathrm{C}-\mathrm{N}$ coupling reaction. TEA, besides being advantageous in terms of cost and mildness, was also uniquely efficacious as a base. Several stronger bases that had been reported to facilitate Pdcatalyzed amination reactions, including DBU and 7-methyl1,5,7-triazabicyclo[4.4.0]dec-5-ene (MTBD), were essentially unable to promote our Ni-catalyzed transformation. Other alkylamine bases, such as 1,4-diazabicyclo[2.2.2] octane (DABCO) and DIPEA, could be used instead of TEA, but with lower reaction yields.
Table 1. Comparison of ligands and bases in the Ni-catalyzed cross-coupling of phenyl triflate (1) and aniline.

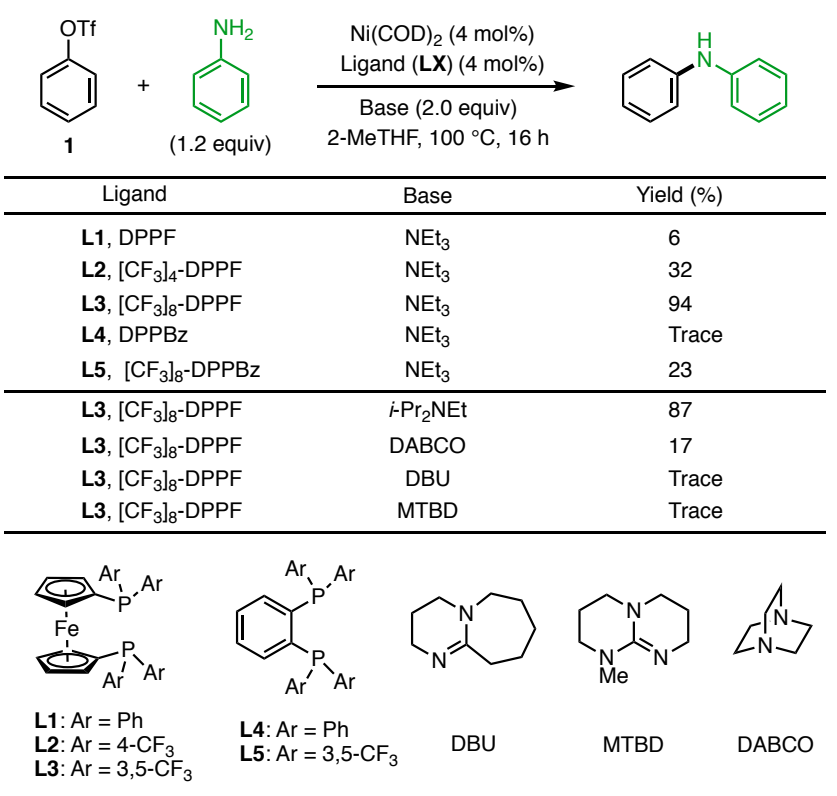

${ }^{a} \mathrm{GC}$ yields were determined relative to hexamethylbenzene internal standard and are reported as a single run. Reaction conditions: phenyl triflate $(0.20 \mathrm{mmol})$, aniline $(0.24 \mathrm{mmol})$, base $(0.40 \mathrm{mmol}), \mathrm{Ni}(\mathrm{COD})_{2}(0.016 \mathrm{mmol}, 4 \mathrm{~mol} \% \mathrm{Ni})$, ligand (0.016 mmol, $4 \mathrm{~mol} \%)$, and 2-MeTHF (0.40 mL, 0.50 M). 2-MeTHF = 2-methyltetrahydrofuran.
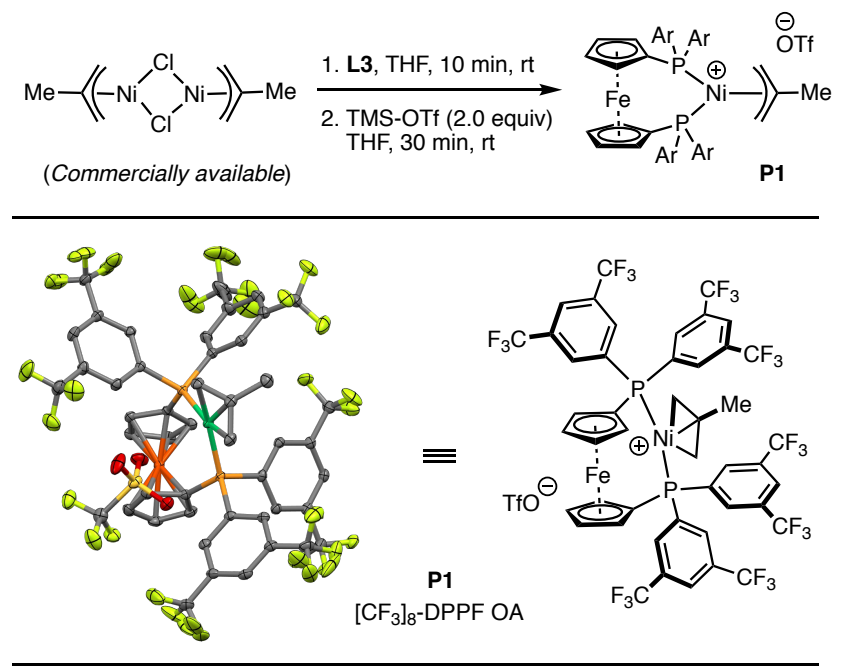

Figure 2. Synthesis and crystal structure of an L3-bound methallyl triflate nickel oxidative addition complex. Thermal ellipsoids are shown at $50 \%$ probability. Hydrogen atoms are omitted for clarity.

Although $\mathrm{Ni}(\mathrm{COD})_{2}$ is a convenient source of $\mathrm{Ni}(0)$ for reaction discovery and mechanistic studies, the complex is highly sensitive to air and moisture, generally requiring the use of an inert atmosphere glovebox to handle. ${ }^{23}$ To alleviate the associated operational complications, we aimed to develop an air-stable Ni precatalyst bearing $\mathbf{L 3}$, the most effective ligand. ${ }^{24}$ 
Table 2. Amination of Aryl Triflates using $\mathbf{P 1}{ }^{a}$

$$
\text { (1.2 equiv) }
$$

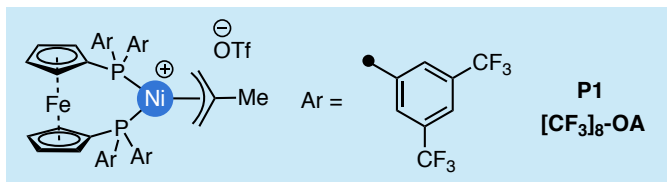<smiles>COC(=O)c1ccccc1Nc1ccnc(C(F)(F)F)c1</smiles>

$2 \mathbf{2 a}$<smiles>N#Cc1ccc(N2CCc3ccccc32)c(F)c1</smiles><smiles>COC(=O)c1cccc(Nc2ccccc2Cc2ccccc2)c1</smiles>

$2 \mathrm{~g}$
$88 \%$

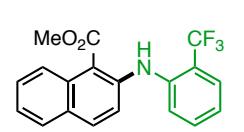

2b<smiles>COC(=O)c1sccc1Nc1cccc(C#N)c1</smiles>

$2 c$
$94 \%$<smiles>Cc1cccc(Nc2ccc(/N=N/c3ccccc3)cc2)c1[Na]</smiles>

$\mathbf{2 d}$
$88 \%$<smiles>COc1cc(Nc2ccnc3c(C(F)(F)F)cccc23)cc(OC)c1</smiles>

$2 e$
$82 \%$<smiles>CC(=O)c1ccc(Nc2c(F)cccc2F)cc1</smiles>

$\stackrel{2 f}{2 f}{ }^{b}$

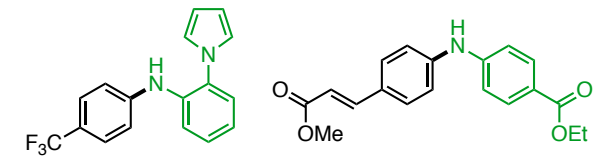

$\underset{95 \%}{\mathbf{2 i}}$

$\stackrel{2 \mathbf{j}}{81 \%}$

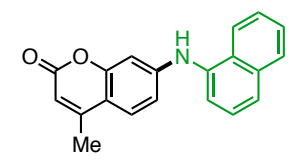

$\mathbf{2 k}$
$89 \%$

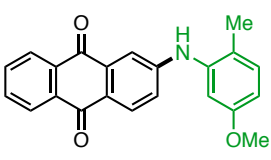

21
$86 \%$<smiles>Cc1cc(Nc2ccccc2N2CCOCC2)ccc1S(C)(=O)=O</smiles>
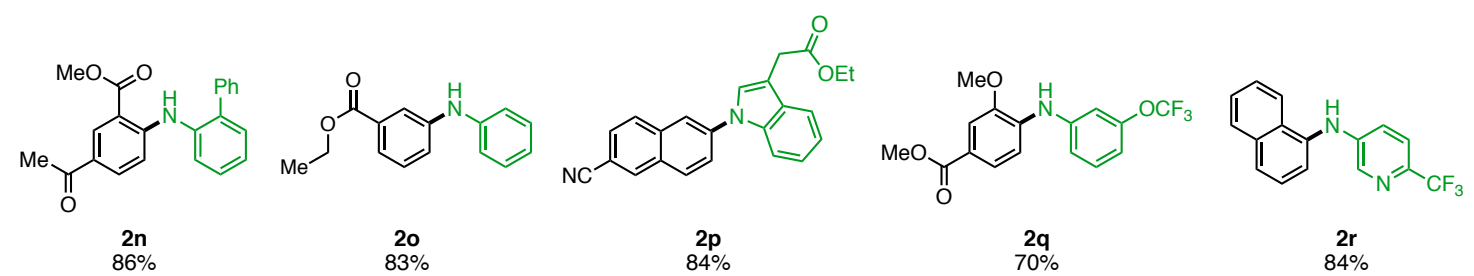

20
$83 \%$

$84 \%$

$2 r$
$84 \%$

${ }^{a}$ Isolated yields are reported as the average of two runs. Unless noted, standard reaction conditions: aryl triflate (1.0 mmol), aryl amine $(1.2 \mathrm{mmol})$, triethylamine $(2.0 \mathrm{mmol}), \mathbf{P 1}(0.04 \mathrm{mmol}, 4 \% \mathrm{Ni}), 2-\mathrm{MeTHF}(2.0 \mathrm{~mL}, 0.5 \mathrm{M}), 100{ }^{\circ} \mathrm{C}$ for $16 \mathrm{~h}$. ${ }^{\mathrm{b}} \mathrm{Single} \mathrm{reaction}$ performed without stirring. ${ }^{\mathrm{c}} 1.5$ equiv of aryl amine was used.

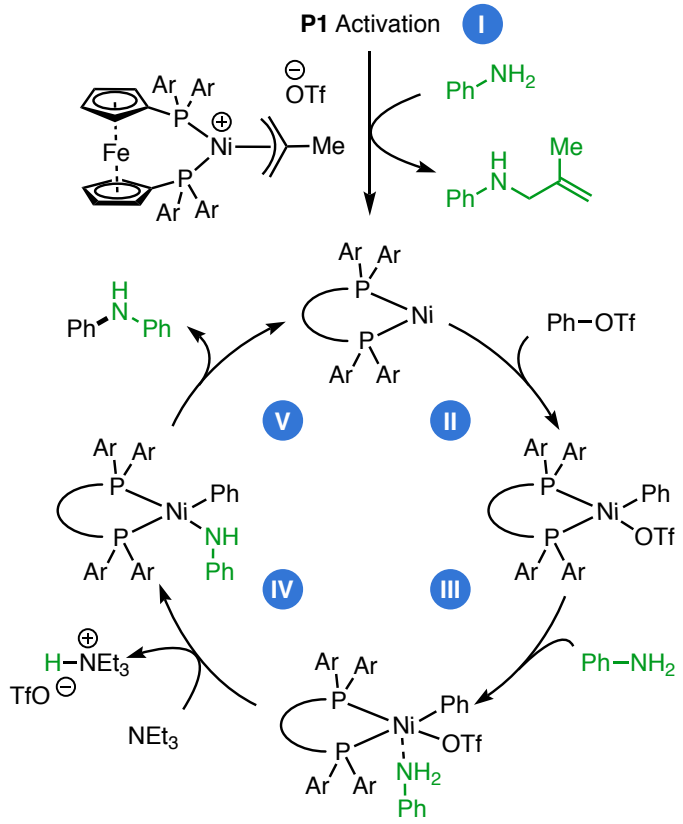

Figure 3. The proposed catalytic cycle for the nickelcatalyzed cross-coupling of aryl halides with anilines: I, activation of P1; II, oxidative addition of an aryl triflate; III, aniline binding to OA complex; IV, deprotonation of an amine-bound OA complex; $\mathbf{V}$, reductive elimination of an amido complex.
Our initial efforts focused on the use of $\sigma$-aryl "oxidative addition" (OA) complexes of aryl bromides and chlorides. ${ }^{25}$ However, OA complexes bearing $\mathbf{L 3}$ and various aryl groups, ${ }^{26}$ including $o$-tolyl and mesityl, were unable to facilitate the reaction, even when activated with reducing additives including phenylboronic acid and activated olefins. We hypothesized that the presence of strongly associating halide anions inhibits $\mathrm{C}-\mathrm{N}$ coupling by outcompeting aniline for binding to $\mathrm{Ni}$ (see below for further mechanistic discussion). ${ }^{27}$ Predicated on this lack of reactivity, we sought to prepare OA complexes bearing noncoordinating triflate anions. ${ }^{28}$ However, due to the propensity of coordinatively unsaturated $\mathrm{Ni}$ (II) complexes to undergo bimetallic decomposition pathways, our attempts to isolate $\mathrm{Ni}(\mathrm{II}) \sigma$-aryl complexes bearing triflate anions were not successful. Based on the work of Nolan ${ }^{29}$ and Hazari, ${ }^{30}$ we hypothesized that the introduction of an $\eta^{3}$-allyl group would saturate the $\mathrm{Ni}$ coordination sphere without introducing new strongly-coordinating ligands such as halides. Combining a commercially available methallyl nickel chloride dimer with $\mathbf{L 3}$ in the presence of THF led to the formation of $\mathbf{L 3}-\mathrm{Ni}(\mathrm{Cl})\left(\eta^{3}\right.$-methallyl). ${ }^{31}$ This complex was not purified, but immediately treated with trimethylsilyl triflate (TMS-OTf), upon which a methallyl nickel triflate complex was rapidly formed. ${ }^{32}$ The structure of this complex (P1) was unambiguously characterized using X-ray diffraction (Figure 2). Under optimized reaction conditions, this precatalyst (P1) facilitated the $\mathrm{C}-\mathrm{N}$ coupling reaction and provided the desired product in 

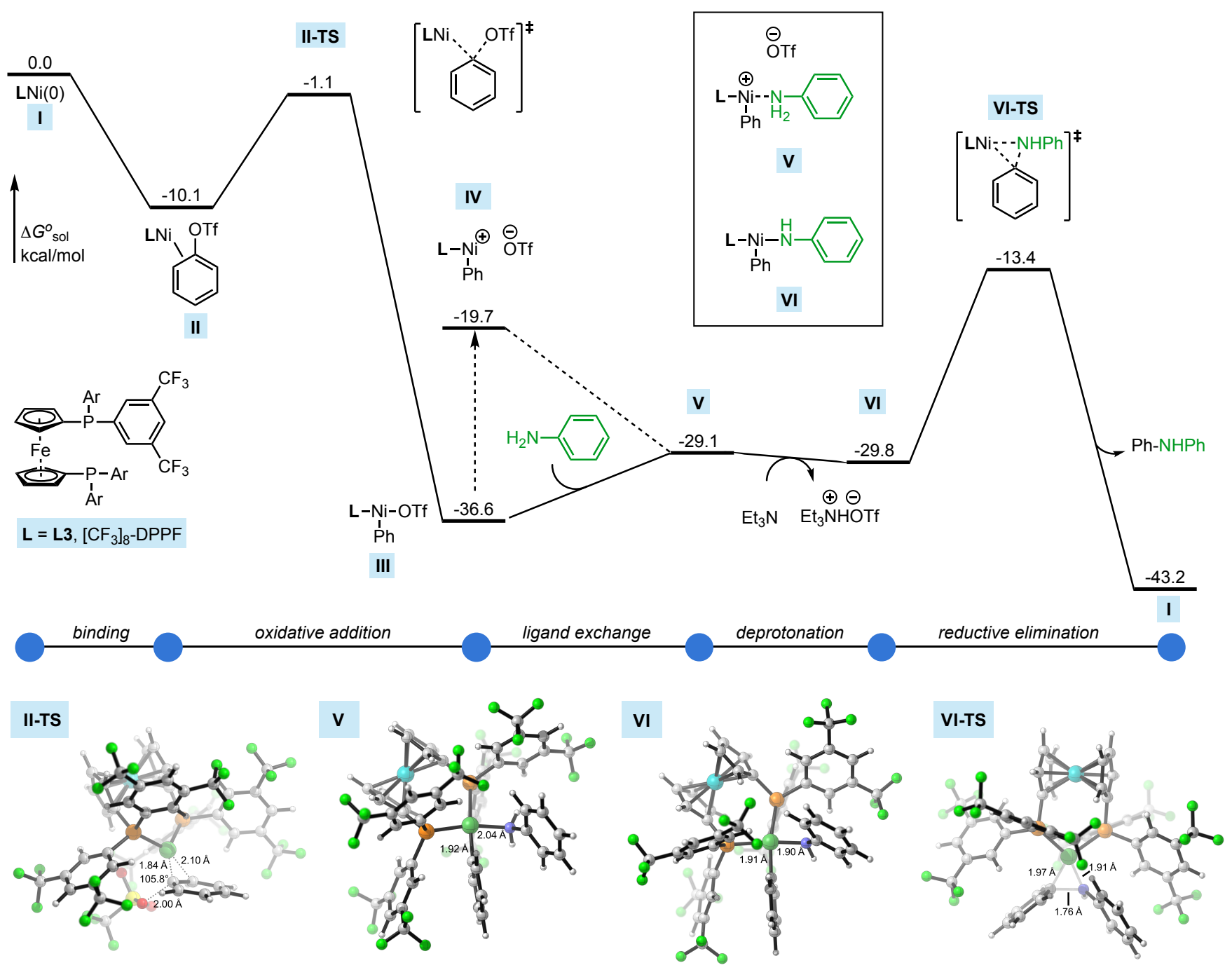

Figure 4. Computed energy profiles for the Ni-catalyzed cross-coupling of $\mathbf{1}$ and aniline. Gibbs free energy values calculated with M06/6-311+G(d,p)-SDD(Ni,Fe)//B3LYP/6-31G(d)-SDD(Ni,Fe).

$98 \%$ yield in $2 \mathrm{~h}$. Analysis of the crude reaction mixture (GC/MS) showed that $N$-methallyl aniline was formed during the reaction, consistent with activation of $\mathbf{P 1}$ through outersphere nucleophilic attack by aniline at the methallyl ligand. ${ }^{33}$

Using this new precatalyst, we explored the scope of the cross-coupling reaction by testing a variety of aryl triflate electrophiles and amine nucleophiles. In contrast to some Pdcatalyzed amination procedures, in particular those facilitated by soluble organic bases, this methodology is tolerant of sterically encumbered coupling partners. Specifically, aryl triflates and anilines bearing bulky ortho-substituents, such as trifluoromethyl (2b), benzyl (2h), morpholino (2m) and phenyl (2n), underwent coupling in high yields. In contrast to traditional Ni-catalyzed amination protocols that work well for strongly coordinating alkylamine nucleophiles, the current method is especially effective for weakly coordinating anilines, including those bearing cyano (2c), trifluoromethoxy (2q), and carbonyl substituents (2a, $\mathbf{2 j}$ ). Additionally, secondary aryl amines, including indoline (2g) and a 3-substituted indole (2p) could be arylated in high yields. ${ }^{22}$ We note, however, that aliphatic amines do not react under these conditions, likely due to their decreased acidity compared to anilines. ${ }^{34}$ Coupling partners containing heterocycles, including pyridines (2a, 2r), a quinoline (2e), a thiophene (2c), and a pyrrole (2i) were tolerated well. Several electrophilic functional groups, including methyl esters (2a-c, 2 h, $2 \mathbf{n})$ and nitriles (2c, 2g, 2p), remained intact under the mildly basic reaction conditions. An $\alpha, \beta$-unsaturated ester $(\mathbf{2} \mathbf{j})$, and a coumarin derivative ( $\mathbf{2} \mathbf{k})$ could be cross-coupled under these reaction conditions, despite their potential to react with anilines in metal-catalyzed aza-Michael reactions. $^{35}$ Moreover, substrates bearing redox-sensitive functional groups, including an anthraquinone (2l) are tolerated. $^{36}$ Finally, because reproducibility issues associated with stirring rate can occur in amination protocols featuring inorganic bases $^{37}$ or electric potentials, ${ }^{18 b}$ we wished to demonstrate that this method is not dependent on mixing efficiency. To show this, we prepared $\mathbf{2 f}$ without using a stir bar or external agitation. The desired product was obtained in $93 \%$ yield, which is in line with that obtained when magnetic stirring was used. This result suggests that these single-phase reactions are less prone to reproducibility issues when varied stirring techniques are used. 
Table 3. Ligand effects on deprotonation and reductive elimination. $^{a}$

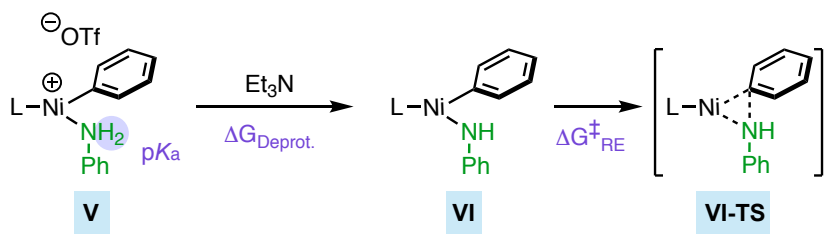

\begin{tabular}{|c|c|c|c|c|c|}
\hline Ligand for $\mathbf{V}$ & $\begin{array}{c}\mathrm{pK}_{\mathrm{a}} \\
(\mathrm{THF})\end{array}$ & $\begin{array}{l}\Delta \mathrm{G}_{\text {Deprot. }} \\
\text { (kcal/mol) }\end{array}$ & $\begin{array}{c}\Delta \mathrm{G}^{\ddagger} \mathrm{RE} \\
(\mathrm{kcal} / \mathrm{mol})\end{array}$ & $\begin{array}{l}\Delta \mathrm{G}^{\ddagger} \mathrm{RE}+ \\
\Delta \mathrm{GDeprot}\end{array}$ & $\begin{array}{c}\text { Yield } \\
(\%)\end{array}$ \\
\hline L1 & 15.3 & 6.4 & 17.2 & 23.6 & $6 \%$ \\
\hline L2 & 13.4 & 2.0 & 16.7 & 18.7 & $32 \%$ \\
\hline L3 & 12.2 & -0.6 & 16.4 & 15.8 & $94 \%$ \\
\hline L5 & 13.0 & 1.3 & 17.9 & 19.2 & $23 \%$ \\
\hline $\mathrm{PhNH}_{2}$ & \multicolumn{5}{|l|}{28.5} \\
\hline $\mathrm{Et}_{3} \mathrm{~N} \cdot \mathrm{HOTf}$ & \multicolumn{5}{|c|}{12.5 (experimental, see ref. 41 ) } \\
\hline
\end{tabular}
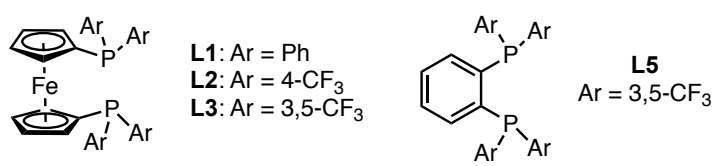

${ }^{a}$ Gibbs free energy values and acidities calculated with M06/6-311+G(d,p)-SDD(Ni,Fe)//B3LYP/6-31G(d)-

$\mathrm{SDD}(\mathrm{Ni}, \mathrm{Fe})$, except for the $\mathrm{p} K_{\mathrm{a}}$ of $\mathrm{Et}_{3} \mathrm{NHOTf}$, used as a reference, which was obtained from the literature. ${ }^{41}$

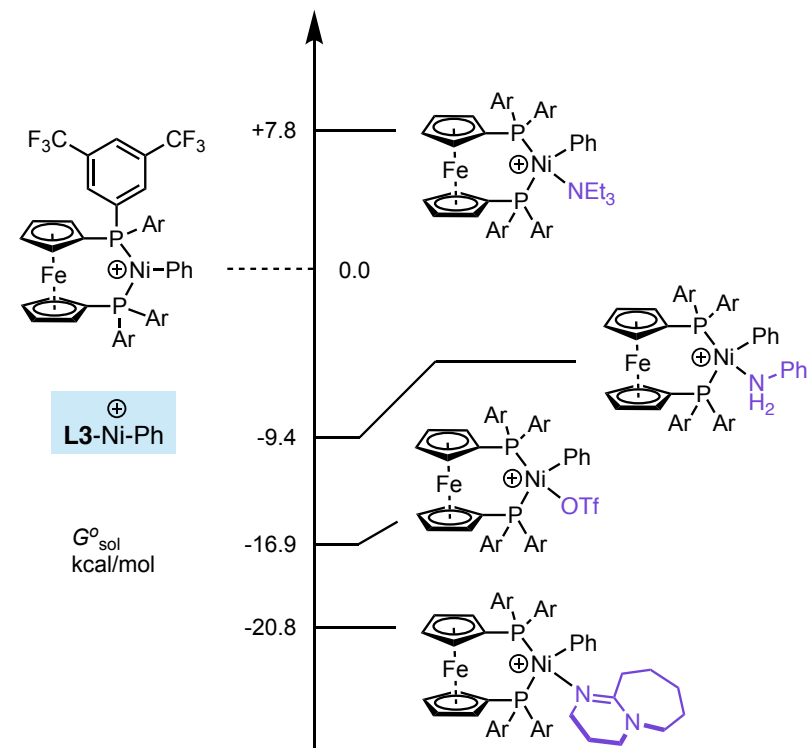

Figure 5. Relative binding energies of triethylamine, triflate anion, and DBU to an L3-supported, cationic nickel complex. Gibbs free energy values calculated with M06/6-311+G(d,p)$\mathrm{SDD}(\mathrm{Ni}, \mathrm{Fe}) / / \mathrm{B} 3 \mathrm{LYP} / 6-31 \mathrm{G}(\mathrm{d})-\mathrm{SDD}(\mathrm{Ni}, \mathrm{Fe})$.

The proposed catalytic cycle of this Ni-catalyzed amination reaction is summarized in Figure $3 .^{20 b, 38}$ First, activation of $\mathbf{P 1}$ via nucleophilic attack of the aniline at the methallyl group provides a $\mathbf{L 3}$-supported $\mathrm{Ni}(0)$ catalyst. Next, $\mathrm{Ni}$ undergoes oxidative insertion into the aryl triflate. Then, the amine binds to the Lewis acidic Ni(II) metal center, acidifying its hydrogens for deprotonation by TEA. Finally, reductive elimination from resultant $\mathrm{Ni}$ (II)-amido complex affords the desired product and regenerates the $\mathrm{Ni}(0)$ catalyst. Although this proposed mechanism is directly analogous to that of other $\mathrm{Ni}$ - and $\mathrm{Pd}$ - catalyzed $\mathrm{C}-\mathrm{N}$ cross-coupling reactions, ${ }^{13}$ it was important to determine how the highly fluorinated ligand $\mathbf{L 3}$ might affect the elementary steps, and importantly, how it is able to facilitate the catalytic transformation using such a weak base (TEA).

Using density functional theory (DFT) calculations we obtained a model of the catalytic mechanism using phenyl triflate (1) and aniline as substrates. The energy profile of this mechanism is illustrated in Figure 4. The binding of phenyl triflate to $\mathbf{L 3}-\mathrm{Ni}(0)$ (complex I) was found to be exergonic by $10.1 \mathrm{kcal} / \mathrm{mol}$ (II). From this $\pi$-complex, oxidative addition through an $\mathrm{S}_{\mathrm{N}}$ Ar-type mechanism ${ }^{39}$ was predicted to be extremely rapid, with a barrier of only $9.0 \mathrm{kcal} / \mathrm{mol}$ (II-TS), and thermodynamically favorable, releasing $26.5 \mathrm{kcal} / \mathrm{mol}$ of free energy (III). In the ground state of the resultant $\mathrm{Ni}$ (II) complex III, the triflate anion was bound to $\mathrm{Ni}$, although its dissociation appeared possible under the reaction conditions (+16.9 $\mathrm{kcal} / \mathrm{mol}$, IV). Regardless, the displacement of the triflate ligand by aniline is only slightly endergonic $(+7.5 \mathrm{kcal} / \mathrm{mol}, \mathbf{V})$. Interestingly, deprotonation of this cationic $\mathrm{Ni}$-aniline complex by TEA was predicted to be slightly favorable in free energy ($0.7 \mathrm{kcal} / \mathrm{mol}, \mathbf{V I})$. Reductive elimination from this amido complex through a three-membered transition state $(+16.4$ $\mathrm{kcal} / \mathrm{mol}$, VI-TS) would then provide the diphenylamine product.

Considering our original hypothesis that the electrondeficiency of the ligand had a favorable influence on the thermodynamics of the deprotonation step, we more closely examined the effect of varying the phosphine ligand on this process. Table 3 shows the $\mathrm{p} K_{\mathrm{a}}$ of several amine-bound $\mathrm{Ni}(\mathrm{II})$ complexes analogous to Vas well as triethylammonium triflate and aniline for comparison purposes. The free energy change associated with the proton transfer step can be calculated on the basis of $\mathrm{p} K_{\mathrm{a}}$ differences. The deprotonation of weakly acidic aniline $\left(\mathrm{p} K_{\mathrm{a}}=28\right)$ by triethylamine $\left(\mathrm{p} K_{\mathrm{aH}+}=12.5\right)^{41}$ is thermodynamically highly disfavored. However, association of the aniline to cationic $\mathrm{Ni}$ (II) results in dramatic acidification, to the extent of roughly $13 \mathrm{p} K_{\mathrm{a}}$ units in THF when the ligand is DPPF (L1). With the addition of electron-withdrawing groups on the ligand, the amine is further acidified. Indeed, in the complex with $\mathbf{L 3}$, the aniline is sufficiently activated that it is predicted to be more acidic $\left(\mathrm{p} K_{\mathrm{a}}=12.2\right)$ than triethylammonium triflate. Thus, deprotonation by triethylamine is in this case slightly thermodynamically favorable.

We also found that the barrier to reductive elimination is also somewhat affected by the electronic properties of the phosphine ligand: as the number of trifluoromethyl substituents on the catalyst increase, the reductive elimination is increasingly facile. For comparison, we also evaluated an analogue derived of $\mathbf{L 3}$ from DPPBz (L4). With the L4-ligated catalyst, the free energy of deprotonation and barrier to reductive elimination were both higher $(+1.9 \mathrm{kcal} / \mathrm{mol}$ and +1.5 $\mathrm{kcal} / \mathrm{mol}$, respectively) than from the $\mathbf{L} 3$-bound complexes. Thus, not only the identity of the $P$-aryl groups, but the backbone structure of the chelating ligand significantly influences these steps. The combined barrier from deprotonation-reductive elimination sequence is also shown in 
Table 3 . The net activation energies are qualitatively consistent with the experimentally determined yields using these catalysts.

Finally, our model also explained the superior performance of triethylamine compared to other organic bases, even those that were significantly stronger bases. Previously, in experimental $^{13 \mathrm{a}}$ and theoretical ${ }^{13 \mathrm{~b}}$ mechanistic studies of Pdcatalyzed amination using $\mathrm{DBU}$, we found that off-cycle binding of the base to Pd could have an inhibitory effect. We investigated the relative binding ability of TEA, DBU, and aniline to the cationic intermediate IV (Figure 5). As a reference, we had found earlier that the binding of triflate to IV is exergonic by $16.9 \mathrm{kcal} / \mathrm{mol}$. Due presumably to steric interactions, the binding of TEA to $\mathbf{I V}$ is significantly disfavored $\left(\Delta \mathrm{G}^{\circ}=+7.8 \mathrm{kcal} / \mathrm{mol}\right)$, in a manner similar to wellknown "frustrated" Lewis acid-base pairs. ${ }^{40}$ Accordingly, aniline can outcompete the base for binding $\left(\Delta \mathrm{G}^{\circ}=-9.4\right.$ $\mathrm{kcal} / \mathrm{mol}$ for aniline binding to $\mathbf{I V}$ ), and the productive reaction can take place. In contrast, when DBU is present, we found that it tightly coordinates to IV $\left(\Delta \mathrm{G}^{\circ}=-20.8 \mathrm{kcal} / \mathrm{mol}\right)$, sequestering $\mathrm{Ni}$ in this off-cycle resting state and thus increasing the overall activation energy for cross-coupling. We believe that this effect explains the unique effectiveness of TEA compared to stronger, more nucleophilic organic bases.

\section{CONCLUSION}

In summary, we have developed a novel $\mathrm{Ni}$ (II) precatalyst bearing an electron-deficient DPPF-derived ligand (L3) that is able to facilitate the cross-coupling of aryl triflates with primary anilines, as well as indolines and indoles. The precatalyst is a rare example of a bench-stable cationic Ni(II) triflate complex and represents a new class of halide-free $\mathrm{Ni}$ precatalysts that might have general applicability to reactions currently requiring $\mathrm{Ni}(\mathrm{COD})_{2}$. Using DFT calculations, relationships between ligand structure and the energetics of the key deprotonation and reductive elimination steps were elucidated. Moreover, we determined that the unique effectiveness of alkylamine bases can be attributed to their steric bulk, which prevents unwanted binding to cationic Ni intermediates. We anticipate that these mechanistic insights can assist in the development of new Ni-catalyzed cross-coupling methodologies that employ soluble organic bases.

\section{ASSOCIATED CONTENT}

\section{Supporting Information}

Experimental procedures; computational, spectral, and reaction optimization data; X-ray data.

\section{AUTHOR INFORMATION}

\section{Corresponding Author}

sbuchwal@mit.edu

\section{Author Contributions}

${ }^{\dagger}$ R.Y.L. and J.M.D contributed equally to this work.

\section{ACKNOWLEDGMENT}

Research reported in this publication was supported by the National Institutes of Health (R35GM122483) and the NSF Graduate Research Fellowship Program under Grant No. 1122374 (J.M.D.). Any opinions, findings, conclusions, or recommendations expressed in this material are those of the authors and do not necessarily reflect the views of the NIH or NSF. The authors thank Dr. Frieda Zhang for her assistance in elucidating the substrate scope of this methodology and Jacob Rodriguez for assistance with mass spectroscopy experiments. We acknowledge Dr. James Bour for helpful discussions throughout the duration of this study and Dr. Peter Müller for crystallographic analysis. We also thank Dr. Scott McCann, Dr. Alexander Schuppe, and Dr. Christine Nguyen for their assistance in the preparation of this manuscript. 


\section{REFERENCES}

(1) Ruiz-Castillo, P.; Buchwald, S. L. Applications of Palladium-Catalyzed C-N Cross-Coupling Reactions. Chem. Rev. 2016, 116, 12564-12649.

(2) Beletskaya, I. P.; Cheprakov, A. V. The Complementary Competitors: Palladium and Copper in C-N Cross-Coupling Reactions. Organometallics 2012, 31, 7753-7808.

(3) For recent examples, see: (a) (b) Xu, J.; Liu, R. Y.; Yeung, C. S.; Buchwald, S. L. Monophosphine Ligands Promote Pd-Catalyzed C-S Cross-Coupling Reactions at Room Temperature with Soluble Bases. ACS Catalysis 2019, 9, 6461-6466. (b) Laffoon, S. D.; Chan, V. S. Fickes, M. G.; Kotechki, B.; Ickes, A. R. Henle, J.; Napolitano, J. G.; Franczyk, T. S.; Dunn, T. B.; Barnes, D. M.; Haight, A. R.; Henry, R. F. Shekhar, S. Pd-Catalyzed Cross-Coupling Reactions Promoted by Biaryl Phosphorinane Ligands. ACS Catal. 2019, 9, 11691-11708. (c) For a recent example of a reaction featuring a fully soluble trimethylsilanolate base, see: Delaney, C. P.; Kassel, V. M.; Denmark, S. E. Potassium Trimethylsilanolate Enables Rapid, Homogeneous Suzuki-Miyaura Cross-Coupling of Boronic Esters. ACS Catal. 2020, 10, 73-80.

(a) Brewer, A. C.; Hoffman, P. C.; Martinelli, J. R.; Kobierski, M. E.; Mullane, N.; Robbins, D. Development and Scale-Up of a Continuous Aerobic Oxidative Chan-Lam Coupling. Org. Process Res. Dev. 2019, 23, 1484-1498. (b) Kashani, S. K.; Sullivan, R. J.; Andersen, M.; Newman, S. G. Overcoming Solid Handling Issues in Continuous Flow Substitution Reactions Through Ionic Liquid Formation. Green Chem. 2018, 20, 1748-1753.

For an example of this, see: Sperry, J. B.; Price Wiglesworth, K. E.; Edmonds, I.; Fiore, P.; Boyles, D. C.; Damon, D. B.; Dorow, R. L.; Piatnitski Chekler, E. L.; Langille, J.; Coe, J. W. Kiloscale Buchwald-Hartwig Amination: Optimized Coupling of Base-Sensitive 6-Bromoisoquinoline-1-carbonitrile with (S)-3-Amino-2-methylpropan-1-ol. Org. Process Res. Dev. 2014, 18, 1752-1758.

(6) (a) Buitrago Santanilla, A.; Christensen, M.; Campeau, L.-C.; Davies, I. W.; Dreher, S. D. P 2 Et Phosphazene: A Mild, Functional Group Tolerant Base for Soluble, Room Temperature Pd-Catalyzed C-N, C-O, and C-C Cross-Coupling Reactions. Org. Lett. 2015, 17, 3370-3373. (b) Buitrago Santanilla, A.; Regalado, E. L.; Pereira, T.; Shevlin, M.; Bateman, K.; Campeau, L.-C.; Schneeweis, J.; Berritt, S.; Shi, Z.-C.; Nantermet, P.; Liu, Y.; Helmy, R.; Welch, C. J.; Vachal, P.; Davies, I. W.; Cernak, T.; Dreher, S. D. Nanomole-Scale High-Throughput Chemistry for the Synthesis of Complex Molecules. Science 2015, 347, 49-53. (c) Ahneman, D. T.; Estrada, J. G.; Lin, S.; Dreher, S. D.; Doyle, A. G. Predicting Reaction Performance in $\mathrm{C}-\mathrm{N}$ Cross-Coupling Using Machine Learning. Science 2018, 360, 186-190. (d) Gesmundo, N. J.; Sauvagnat, B.; Curran, P. J.; Richards, M. P.; Andrews, C. L.; Dandliker, P. J.; Cernak, T. Nanoscale Synthesis and Affinity Ranking. Nature 2018, 557, 228-232. (e) Uehling, M. R.; King, R. P.; Krska, S. W.; Cernak, T. Buchwald, S. L. Pharmaceutical Diversification via Palladium Oxidative Addition Complexes. Science 2019, 363, 405-408.

(7) (a) Tundel, R. E.; Anderson, K. W.; Buchwald, S. L. Expedited Palladium-Catalyzed Amination of Aryl Nonaflates through the Use of Microwave-Irradiation and Soluble Organic Amine Bases. J. Org. Chem. 2006, 71, 430-433. (b) Beutner, G. L. Coombs, J. R.; Green, R. A.; Inankur, B.; Lin, D.; Qiu, J.;Roberts, F.; Simmons, E. M.; Wisniewski, S. R. Palladium-Catalyzed Amidation and Amination of (Hetero)aryl Chlorides under Homogeneous Conditions Enabled by a Soluble DBU/NaTFA Dual-Base System. Org. Process Res. Dev. 2019, 23, 1529-1537. (c) Kashani, S. K.; Jessiman, J. E.; Newman, S. Exploring Homogeneous Conditions for Mild Buchwald-Hartwig Amination in Batch and Flow, 2019. https://doi.org/10.26434/chemrxiv.10094048.v1.

(8) Murthy Bandaru, S. S.; Bhilare, S.; Chrysochos, N.; Gayakhe, V.; Trentin, I.; Schulzke, C.; Kapdi, A. R. Pd/PTABS: Catalyst for Room Temperature Amination of Heteroarenes. Org. Lett. 2018, 20, 473-476.

(9) (a) Liu, L.; Frohn, M.; Xi, N.; Dominguez, C.; Hungate, R.; Reider, P. J. A Soluble Base for the Copper-Catalyzed Imidazole N-Arylations with Aryl Halides. J. Org. Chem. 2005, 70, 10135-10138. (b) Sung, S.; Sale, D.; Braddock, D. C.; Armstrong, A. Brennan, C.; Davies, R. P. Mechanstic Studies on the Copper-Catalyzed N-Arylation of Alkylamines Promoted by Soluble Ionic Bases. ACS Catal. 2016, 6, 3965-3974. (c) Lo, Q. A.; Sale, D.; Braddock, D. 
C.; Davies, R. P. Mechanistic and Performance Studies on the Ligand-Promoted Ullmann Amination Reaction. ACS Catal. 2018, 8, 101-109.

(10) Morgenthaler, M.; Schweizer, E.; Hoffmann-Röder, A.; Benini, F.; Martin, R. E.; Jaeschke, G.; Wagner, B.; Fischer, H.; Bendels, S.; Zimmerli, D.; Schneider, J.; Diederich, F.; Kansy, F.; Müller, K. Predicting and Tuning Physicochemical Properties in Lead Optimization: Amine Basicities. ChemMedChem 2007, 2, 1100-1115.

(11) Roose, P.; Eller, K.; Henkes, E.; Rossbacher, Höke, H. Ullmann's Encyclopedia of Industrial Chemistry. In Amines, Aliphatic, Wiley, Hoboken, 2015.

(a) Dennis, J. M.; White, N. A.; Liu, R. Y.; Buchwald, S. L. Breaking the Base Barrier: An Electron-Deficient Palladium Catalyst Enables the Use of a Common Soluble Base in C-N Coupling. J. Am. Chem. Soc. 2018, 140, 4721-4725. (b) For additional applications of this catalyst system, see: Engl, P. S.; Häring, A. P.; Berger, F.; Berger, G.; Pérez-Bitrián, A.; Ritter, T. C-N Cross-Couplings for Site-Selective Late-Stage Diversification via Aryl Sulfonium Salts. J. Am. Chem. Soc. 2019, 141, 13346-13351.

(13) (a) Dennis, J. M.; White, N. A.; Liu, R. Y.; Buchwald, S. L. Pd- Catalyzed C-N Coupling Reactions Facilitated by Organic Bases: Mechanistic Investigation Leads to Enhanced Reactivity in the Arylation of Weakly Binding Amines. ACS Catal. 2019, 9, 3822-3830. For computational mechanistic studies, see: (b) Kim, S.-T.; Pudasaini, B.; Baik, M.H. Mechanism of Palladium-Catalyzed $\mathrm{C}-\mathrm{N}$ Coupling with 1,8-Diazabicyclo[5.4.0]undec-7-ene (DBU) as a Base. ACS Catal. 2019, 9, 6851-6856. (c) Sunesson, Y.; Limé, E.; Nilsson Lill, S. O.; Meadows, R. E.; Norrby, P.-O. Role of the Base in Buchwald-Hartwig Amination. J. Org. Chem. 2014, 79, 11961-11969.

(14) Baumgartner, L. M.; Dennis, J. M.; White, N. A.; Buchwald, S. L.; Jensen, K. F. Use of a Droplet Platform To Optimize Pd-Catalyzed C-N Coupling Reactions Promoted by Organic Bases. Org. Process Res. Dev. 2019, 23, $1594-1601$.

(15) (a) Christau, H. J.; Desmurs, J. R. Arylation of Hard Heteroatomic Nucleophiles Using Bromoarenes Substrates and $\mathrm{Cu}, \mathrm{Ni}$, Pd-Catalysts. Ind. Chem. Libr. 1995, 7, 240. For the first general method, see: (b) Wolfe, J. P.; Buchwald, S. L. Nickel-Catalyzed Amination of Aryl Chlorides. J. Am. Chem. Soc. 1997, 119, 6054-6058. (b) For a detailed history of this topic, see: Marín, M.; Rama, R. J.; Nicasio, M. C. Ni-Catalyzed Amination Reactions: An Overview. Chem. Rec. 2016, 16, 1819-1832.

(16) For recent examples, see: (a) Lavoie, C. M.; Tassone, J. P.; Ferguson, M. J.; Zhou, Y.; Johnson, E. R.; Stradiotto, M. Probing the Influence of PAd-DalPhos Ancillary Ligand Structure on Nickel-Catalyzed Ammonia Cross-Coupling. Organometallics, 2018, 37, 4015-4023. (b) McGuire, R. T.; Paffile, J. F. J.; Zhou, Y.; Stradiotto, M. Nickel-Catalyzed C-N Cross-Coupling of Ammonia, (Hetero)anilines, and Indoles with Activated (Hetero)aryl Chlorides Enabled by Ligand Design. ACS Catal., 2019, 9, 9292-9297. For a thorough review on this topic in regard to bisphosphines, see: (c) Lavoie, C. M.; Stradiotto, M. Bisphosphines: A Prominent Ancillary Ligand Class for Application in NickelCatalyzed C-N Cross-Coupling. ACS Catal. 2018, 8, 7228-7250.

(17) (a) Corcoran, E. B.; Pirnot, M. T.; Lin, S.; Dreher, S. D.; DiRocco, D. A.; Davies, I. W.; Buchwald, S. L.; MacMillan, D. W. C. Aryl Amination Using Ligand-Free Ni(II) Salts and Photoredox Catalysis. Science 2016, 353, 279-283. (b) Kudisch, M.; Lim, C.-H.; Thordarson, P.; Miyake, G. M. Energy Transfer to Ni-Amine Complexes in Dual Catalytic, Light-Driven C-N Cross-Coupling reactions. J. Am. Chem. Soc. 2019, 141, 19479-19486. (c) Lim, C.-H.; Kudisch, M.; Liu, B. Miyake, G. M. C-N Cross-Coupling via Photoexcitation of Nickel-Amine Complexes. J. Am. Chem. Soc. 2018, 140, 7667-7673. (d) Park, B. Y.; Pirnot, M. T.; Buchwald, S. L. Visible Light-Mediated (Hetero)aryl Amination Using Ni(II) Salts and Photoredox Catalysis in Flow: A Synthesis of Tetracaine. J. Org. Chem. 2020, 10.1021/acs.joc.9b03107.

(18) (a) Li, C.; Kawamata, Y.; Nakamura, H.; Vantourout, J. C.; Liu, Z.; Hou, Q.; Bao, D.; Starr, J. T.; Chen, J.; Yan, M.; Baran, P. S. Angew. Chem. Int. Ed. 2017, 56, 13088-13093. (b) Kawamata, Y.; Vantourout, J. C.; Hickey, D. P.; Bai, P.; Chen, L.; Hou, Q.; Qiao, W.; Barman, K.; Edwards, M. A.; Garrido-Castro, A. F.; deGruyter, J. N.; Nakamura, H.; Knouse, K.; Qin, C.; Clay, K. J.; Bao, D.; Li, C.; Starr, J. T.; Garcia-Irizarry, C.; Sach, N.; White, H. S.; Neurock, M.; Minteer, S. D.; Baran, P. S. Electrochemically Driven, Ni-Catalyzed Aryl Amination: Scope, Mechanism, and Applications. J. Am. Chem. Soc. 2019, 141, 6392-6402.

(19) (a) Wiensch, E. M.; Montgomery, J. Nickel-Catalyzed Amination of Silyloxyarenes through C-O Bond Activation. Angew. Chem. Int. Ed. 2018, 57, 11045-11049. (b) Harada, T.; Ueda, Y.; Iwai, T.; Sawamura, M. NickelCatalyzed Amination of Aryl Fluoride with Primary Amines. Chem. Commun. 2018, 54, 1718-1721. 
(20) (a) Borzenko, A.; Rotta-Loria, N. L.; MacQueen, P. M.; Lavoie, C. M. McDonald, R.; Stradiotto, M. Nickel-Catalyzed Monoarylation of Ammonia. Angew. Chem. Int. Ed., 2015, 54, 3773-3777. (b) Ge, S.; Green, R. A.; Hartwig, J. F. Controlling First-Row Catalysts: Amination of Aryl and Heteroaryl Chlorides and Bromides with Primary Aliphatic Amines Catalyzed by a BINAP-Ligated Single-Component Ni(0) Complex. J. Am. Chem. Soc. 2014, 136, 1617-1627.

(21) For discussion on the preparation and use of DPPF derivatives in Ni-catalyzed amination reactions, see: McGuire, R. T.; Clark, J. S. K. Gatien, A. V.; Shen, M. Y.; Ferguson, M. J.; Stradiotto, M. Bulky 1,1'-Ferrocenyl Ligands Featuring Diazaphospholene or Dioxaphosphepine Donor Fragments: Catalytic Screening in Nickel-Catalyzed C-N CrossCoupling. Eur. J. Inorg. Chem. 2019, 4112-4116.

(22) A Ni-catalyst bearing this ligand has been shown to facilitate the cross-coupling of aryl chlorides with indoles and primary aliphatic amines when $\mathrm{NaO} t$-Bu is used as the base: Clark, J. S. K.; Voth, C. N.; Ferguson, M. J.; Stradiotto, M. Evaluating 1,1'-Bisphosphinoferrocene Ancillary Ligand Variants in the Nickel-Catalyzed C-N Cross-Coupling of (Hetero)aryl Chlorides. Organometallics 2017, 36, 679-686.

(23) Nattmann, L., Saeb, R., Nöthling, N. et al. An Air-Stable Binary Ni(0)-Olefin Catalyst. Nat Catal. 2019, doi:10.1038/s41929-019-0392-6.

(24) For an overview of Ni precatalysts, see: Hazari, N.; Melvin, P. R.; Mohadjer Beromi, M. Well-Defined Nickel and Palladium Precatalysts for Cross-Coupling. Nat. Rev. Chem. 2017, 1, 25.

(25) For applications of these precatalysts, see: Standley, E. A.; Jamison, T. F. Simplifying Nickel(0) Catalysis: An AirStable Nickel Precatalyst for the Internally Selective Benzylation of Terminal Alkenes. J. Am. Chem. Soc. 2013, 135, $1585-1592$.

For a discussion on the effect of the aryl group on the reactivity of DPPF-ligated $\sigma$-aryl precatalysts, see: Mohadjer Beromi, M.; Banerjee, G.; Brudvig G. W.; Charboneau, D. J.; Hazari, N.; Lant, H. M. C.; Mercado, B. Q. Modifications to the Aryl Group of DPPF-Ligated Ni $\sigma$-Aryl Precatalysts: Impact on Speciation and Catalytic Activity in Suzuki-Miyaura Coupling Reactions. Organometallics 2018, 37, 3943-3955.

(27) The addition of halide scavengers, including $\mathrm{AgOTf}_{\text {and }} \mathrm{AgBF}_{4}$, resulted in one turnover of the catalyst followed by catalyst death and the formation of Ni black.

(28) For an example of Pd precatalysts bearing triflate anions, see: DeAngelis, A. J., Gildner, P. G., Chow, R.; Colacot, T. J. Generating Active "L-Pd(0)" Via Neutral or Cationic $\pi$-Allylpalladium Complexes Featuring Biaryl/ Bipyrazolylphosphines: Synthetic, Mechanistic, and Structure-Activity Studies in Challenging Cross-Coupling reactions. J. Org. Chem. 2015, 80, 6794-6813.

(29) Martin, A. R.; Nelson, D. J.; Meiries, S.; Slawin, A. M. Z.; Nolan, S. P. Efficient C-N and C-S Bond Formation Using the Highly Active [Ni(allyl)Cl(IPr*OMe $)$ Precatalyst. Eur. J. Org. Chem. 2014, 3127-3131.

(30) Beromi, M. M.; Banerjee, G.; Brudvig, G. W.; Hazari, N. Mercado, B. Q. Nickel(I) Aryl Species: Synthesis, Properties, and Catalytic Activity. ACS Catal. 2018, 8, 2526-2533.

(31) These catalysts have been shown to be active in other Ni-catalyzed cross-coupling methodologies. See: Ge, S.; Hartwig, J. F. Highly Reactive, Single-Component Nickel Catalyst Precursor for Suzuki- Miyuara Cross-Coupling of Heteroaryl Boronic Acids with Heteroaryl Halides. Angew. Chem. Int. Ed. 2012, 51, 12837-12841

(32) Weber, J. M.; Longstreet, A. R.; Jamison, T. F. Bench-Stable Nickel Precatalysts with Heck-type Activation. Organometallics 2018, 37, 2716-2722

(33) For another example of this reactivity, see: Pawlas, J.; Nakao, Y.; Kawatsura, M.; Hartwig, J. F. A General NickelCatalzyed Hydroaminatino of 1,3-Dienes by Alkylamines: Catalyst Selection, Scope, and Mechanism. J. Am. Chem. Soc. 2002, 124, 3669-3669.

(34) Preliminary results have shown that stronger organic bases, including phosphazene base tert-butyliminotri(pyrrolidino)phosphorane (BTTP), can facilitate the cross-coupling of phenyl triflate and benzyl amine in the presence of $\mathbf{P 1}$. 
(35) For examples of the addition of aniline derivatives to $\alpha, \beta$-unsaturated electrophiles, see: (a) Kim, S.; Kang, S.; Kim, G.; Lee, Y. Copper-Catalyzed Aza-Michael Addition of Aromatic Amines or Aromatic Aza-Heterocycles to $\alpha, \beta-$ Unsaturated Olefins. J. Org. Chem. 2016, 81, 4048-4057. (b) Fei, X.-D.; Zhou, Z.; Li, W.; Zhu, Y.-M.; Shen, J.-K. Buchwald-Hartwig Coupling/Michael Addition Reactions: One-Pot Synthesis of 1,2-Disubstituted 4-Quinolones from Chalcones and Primary Amines. Eur. J. Org. Chem. 2012, 3001-3008.

(36) Bachman, J. E.; Curtiss, L. A.; Assary, R. S. Investigation of the Redox Chemistry of Anthraquinone Derivatives Using Density Functional Theory. J. Phys. Chem. A 2014, 118, 8852-8860.

(37) (a) Meyers, C.; Maes, B. U. W.; Loones, K. T. J.; Bal, G.; Lemière, G. L. F.; Dommisse, R. A. Study of a New Rate Increasing "Base Effect" in the Palladium-Catalyzed Amination of Aryl Iodides. J. Org. Chem. 2004, 69, 6010-6017. (b) Kuethe, J. T.; Childers, K. G.; Humphrey, G. R.; Journet, M.; Peng, Z. A Rapid, Large-Scale Synthesis of a Potent Cholecystokinin (CCK) 1R Receptor Agonist. Org. Process Res. Dev. 2008, 12, 1201-1208.

(38) (a) Uthayopas, C.; Surawatanawong, P. Aryl C-O Oxidative Addition of Phenol Derivatives to Nickel Supported by an $\mathrm{N}$-Heterocyclic Carbene via a Ni ${ }^{0}$ Five-Centered Complex. Dalton Trans. 2019, 48, 7817-7827. (b) Rull, S. G.; Funes-Ardoiz, I.; Maya, C.; Maseras, F.; Fructos, M. R.; Belderrain, T. R.; Nicasio, M. C. Elucidating the Mechanism of Aryl Aminations Mediated by NHC-Supported Nickel Complexes: Evidence for a Nonradical Ni(0)/Ni(II) Pathway. ACS Catal. 2018, 8, 3733-3742. For a discussion on catalytically-active Ni(I) and Ni(II) species, see: (c) Lavoie, C. M.; McDonald, R.; Johnson, E. R.; Stradiotto, M. Bisphosphine-Ligated Nickel Pre-Catalysts in C(sp $\left.{ }^{2}\right)-\mathrm{N}$ Cross-Couplings of Aryl Chlorides: A Comparison of Nickel(I) and Nickel(II). Adv. Synth. Catal. 2017, 359, 29722980 .

(39) Bajo, S.; Laidlaw, G.; Kennedy, A. R.; Sproules, S.; Nelson, D. J. Oxidative Addition of Aryl Electrophiles to a Prototypical Nickel(0) Complex: Mechanism and Structure/Reactivity Relationships. Organometallics 2017, 36, $1662-1672$.

(40) For some recent reviews, see: (a) Stephan, D. W. The Broadening Reach of Frustrated Lewis Pair Chemistry. Science 2016, 354, aaf7229. (b) Stephan, D. W. Frustrated Lewis Pairs: From Concept to Catalysis. Acc. Chem. Res. 2015, 48, 306-316. (c) Stephan, D. W.; Erker, G. Frustrated Lewis Pair Chemistry: Development and Perspectives. Angew. Chem. Int. Ed. 2015, 54, 6400-6441.

(41) Rodima, T.; Kaljurand, I.; Pihl, A.; Maemets, V.; Leito, I.; Koppel, I. A. Acid-Base Equilibria in Nonpolar Media. 2.1 Self-Consistent Basicity Scale in THF Solution Ranging from 2-Methoxypyridine to EtP ${ }_{1}$ (pyrr) Phosphazene. $J$. Org. Chem. 2002, 67, 1873-1881.

\section{TOC Image}

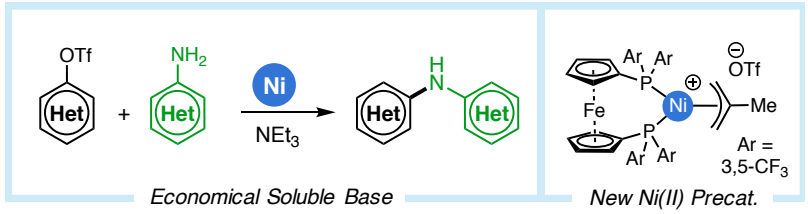

